\title{
Conceitualização e Medida do quanto a Experiência do usuário pode ser Cool: Uma revisão sistemática da literatura
}

\author{
Rosângela Divina de Sousa Santana, Deller James Ferreira, Luciana de Oliveira Berretta \\ Instituto de Informática - Universidade Federal de Goiás (UFG) \\ Goiânia - GO - Brazil \\ (rosousas,deller,luciana.berretta)@ufg.br
}

\begin{abstract}
This article presents a systematic literature review in order to define a more general concept of the term "coolness" and exemplify how it can be operationalized to measure how much the user experience is "cool". The motivation for carrying out this work is that the term has been integrated into the design of computational interfaces and has become part of humancomputer interaction (IHC). Despite the valuable contributions to date, there is still no well-established concept. There is a need to develop a more comprehensive definition and a demand for the creation of an instrument that can determine how cool a computational interface is. Through a systematic literature review, articles that address the term "coolness" were analyzed. In this process, several subconcepts linked to each area were integrated, as well as instruments generated from these subconcepts to measure how legal a digital product is.
\end{abstract}

Resumo. Este artigo apresenta uma revisão sistemática da literatura com objetivo de definir um conceito mais geral do termo "coolness" e exemplificar como ele pode ser operacionalizado para medir o quanto a experiência do usuário é "cool". Em português "coolness" pode ser entendido como o quanto algo pode ser legal. A motivação para a realização deste trabalho é que o termo vem sendo integrado ao projeto de interfaces computacionais e tornado parte da interação humano-computador (IHC). Apesar das valiosas contribuições até o momento, ainda não há um conceito bem estabelecido. Há uma necessidade de se elaborar uma definição mais abrangente e uma demanda para a criação de um instrumento que possa determinar o quanto uma interface computacional é cool, em português legal. Através de uma revisão sistemática da literatura foram analisados artigos publicados que abordam o termo "coolness". Nesse processo foram integralizadas vários sub conceitos vinculados ao conceito de "coolness", assim como instrumentos gerados a partir desses subconceitos para medir o quanto um produto digital élegal.

\section{Introdução}

Atualmente $\mathrm{cool}$, em português "legal", é uma expressão de aprovação muito comum para descrever quase todas as entidades, incluindo objetos, pessoas ou fenômenos [Sundar 2014]. O termo coolness tem sido explorado por pesquisadores na área de 
Interação Humano-Computador (IHC), que buscam subsídios para o projeto de produtos interativos. Fatores psicológicos, como o coolness, são muito importantes e utilizados por profissionais em diversas áreas como: projetistas, desenvolvedores de aplicativos e sistemas e profissionais de marketing, que os aplicam em novos produtos e interfaces.

O conceito coolness está sendo integrado aos processos de projeto interativo e tornado parte do projeto da interação humano-computador [Bruun 2016]. Embora os pesquisadores ainda não concordem com uma definição específica na área de interação humano computador (IHC), do que seja coolness (algo legal) [Sundar 2014] existem alguns atributos específicos que podem ser percebidos pelos usuários e caracterizados como tal.

O termo coolness tem sido principalmente abordado por pesquisadores na área de marketing [Nancarrow 2002], que tentam definir e entender melhor este conceito [O'Donnell 2000], assim como explorá-lo na experiência dos usuários para a criação de produtos mais interessantes [Holtzblatt 2011]. Mas como podemos definir o que é coolness?

Os estudos em áreas como marketing, psicologia e IHC atrelam alguns conceitos ao coolness tais como: emoções, afeto, qualidade hedônica e pragmática da interface, diversão, fluidez, encantamento, dentre outros. Warren et al. (2014) e Price et al. (2016) concordam com quatro propriedades definidoras para o conceito de que coolness é socialmente construído. Pode-se dizer que é uma percepção ou uma atribuição concedida por um público em vez de uma inerente característica de um objeto ou pessoa [Price et al. 2016]. Como popularidade ou status, é semelhante a uma característica socialmente construída sendo altamente relacionado à cultura do indivíduo.

No mercado, o conceito tem sido considerado um catalisador provocador para a venda de produtos, de forma que uma imagem legal ajuda a solidificar o produto, além de contribuir para sentimentos pessoais de realização, conexão com outros, identidade e experiências positivas. Alguns autores produziram questionários com itens identificados no contexto de coolness e fatores relacionados à subcultura, atratividade e originalidade, como uma forma de aferir o quanto um produto é caracterizado como cool.

Apesar das valiosas contribuições até o momento, ainda não há uma definição geral do conceito de coolness e como seus aspectos contribuem para uma boa experiência do usuário (UX) em produtos digitais. Nesse contexto, há uma necessidade de se elaborar uma definição mais ampla do termo coolness e uma demanda para a criação de instrumentos que possam determinar o quanto uma interface é cool.

A realização de uma revisão sistemática sobre o tema supracitado se justifica dada a grande relevância do tema. Além disso, tanto quanto sabemos, não há revisão sistemática nessa perspectiva. Desse modo, é importante a realização de uma revisão sistemática da literatura (SLR) que culmine em um melhor entendimento do termo coolness, assim como ele pode ser operacionalizado para medir a experiência do usuário. Dessa forma, a contribuição desta revisão sistemática é prover uma definição mais ampla, integralizando diferentes aspectos abordados na literatura, além de exemplificar como o conceito pode ser operacionalizado para medir o quanto a experiência do usuário está sendo legal em produtos digitais. 


\section{Importância do conceito de coolness em UX}

Por que a definição de um conceito para o coolness e entendimento de que o cool é relevante para a UX?

Segundo Raptis (2016) e Bruun et al. (2016) na área de IHC são investigadas nossas interações com as tecnologias digitais e são realizadas pesquisas sobre nossas experiências. A experiência do usuário [Hassenzahl and Tractinsky 2006] é um dos principais fluxos de pesquisa da área de IHC, e engloba a ideia de que nossas interações com artefatos digitais devem transcender a eficácia e a eficiência. Porém a UX não se restringe à área de IHC e tampouco a artefatos digitais. A experiência do usuário é de natureza subjetiva, pois é sobre a percepção e pensamento individual no que diz respeito ao uso de um produto, sistema ou serviço específico. É também dinâmica, podendo ser constantemente modificada ao longo do tempo devido à evolução das circunstâncias e inovações.

Na área de IHC a UX envolve aspectos relacionados à eficiência e eficácia, mas, além disso, enfatiza aspectos afetivos e experienciais, significativos e valiosos de interação humano-computador. Um aspecto importante em UX é o processo pelo qual os usuários formam experiências. Quando o usuário encontra um produto, forma uma impressão momentânea, que evolui ao longo do tempo. Neste processo, a percepção, ação, motivação e cognição do usuário se integram para formar uma história memorável e coerente chamada "experiência do usuário". Esse processo suscita respostas emocionais, que determinam em grande parte se a experiência será considerada positiva ou negativa.

A percepção de um produto é baseada no indivíduo, nos valores do usuário e no contexto de uso, portanto, a percepção da experiência do usuário é altamente subjetiva. Além disso, em um mercado competitivo, os valores subjetivos de todas as partes interessadas, como a organização e o usuário, desempenham um papel central no desenvolvimento da experiência do usuário [Jetter and Gerken 2007]. A subjetividade da UX abre margem para a investigação de vários fatores psicológicos, dentre eles a percepção de que algo é cool.

Alguns autores sugerem que o termo foi conceituado inicialmente por guerreiros africanos como forma de se destacarem diante do perigo [Pountain et al. 2000]. Existe ainda o conceito aplicado na contemporaneidade, como característica de contracultura, que tornou-se o meio que pequenos grupos de músicos de jazz negros resistiram contra o branco com sua cultura dominante, englobando uma maneira diferente de se vestir e comportar-se.

Raptis et al. (2013), afirmam que usamos o cool em nossa vida cotidiana para descrever as pessoas, objetos, atividades, e quando atribuímos a palavra cool a um objeto, basicamente percebemos algumas de suas características interessantes ou legais em um momento específico e dentro de um contexto social. Se o contexto mudar, então as mesmas características podem ser percebidas de forma diferente como não legal. Como resultado, há uma interação entre um indivíduo e o objeto, dentro de um contexto social.

A comunidade IHC adotou coolness como um conceito importante e está discutindo suas implicações em UX como demonstrado nos trabalhos de Holtzblatt (2010), Sundar et al. (2014), Raptis et al. (2016) e Bruun et al. (2016). Holtzblatt (2010) 
foi a pioneira a introduzir o coolness na área de IHC e afirma que uma boa experiência do usuário está intimamente ligada à experiência de alegria e consequentemente ao conceito de coolness.

\section{Método}

Este estudo foi realizado por meio de uma revisão sistemática da literatura com base nas diretrizes originais propostas por [Brereton et al. 2007].

"Uma revisão literária sistemática é um meio de identificar, avaliar e interpretar todas as pesquisas disponíveis relevantes a uma determinada questão de pesquisa, ou área de um tópico, ou fenômeno de interesse. Estudos individuais que contribuem para uma revisão sistemática são chamados estudos primários; uma revisão sistemática é uma forma de estudo secundário." [Brereton et al. 2007].

Os passos no método de revisão sistemática da literatura seguidos foram planejamento e execução da pesquisa.

\section{Planejamento da Revisão Sistemática}

No início do planejamento foi detectada a necessidade da realização de uma revisão sistemática, pois não foi encontrada uma revisão sobre o tema proposto. A seguir são apresentadas as fases do planejamento subsequentes a avaliação da necessidade da revisão, que são definição das questões de pesquisa, formulação das strings de busca, seleção das bases de dados e elaboração dos critérios de inclusão e exclusão das fontes bibliográficas.

\subsection{Questões de Pesquisa}

Até o momento, apenas alguns estudos da literatura apresentaram informações sobre o conceito coolness. Esses poucos estudos estão voltados para a identificação das características do coolness em determinada área. Diante desse contexto, para prover uma definição mais ampla, integralizando diferentes aspectos abordados na literatura, buscamos artigos que trataram do termo coolness em periódicos, teses, dissertações publicados no Brasil e no exterior de 2010 até 2020. A realização de uma revisão sistemática ajudará a descrever uma definição geral sobre o conceito coolness e sua instrumentalização em UX.

Com o intuito de apresentar como o conceito de coolness é definido e instrumentalizado na literatura, com o intuito de prover uma visão holística do termo, as questões de pesquisa que norteiam esta revisão sistemática são:

QP1. Do que se trata o conceito de coolness em produtos digitais?

QP2. Como conceito de coolness é operacionalizado para testar o quanto a experiência do usuário está sendo cool em produtos digitais?

\subsection{Seleção das Bases de Dados e Strings de Busca}

Foram realizadas várias combinações de palavras-chave, organizadas em strings de busca utilizando operações booleanas AND e OR. Após a realização de alguns testes 
com strings de busca, foram verificadas quais delas resultaram nas melhores taxas de aceitação e a quantidade de resultados retornados. Diante disso, as palavras chaves selecionadas para a realização das buscas foram: ("consumer coolness" OR "social coolness" OR "coolness attractiveness" OR "coolness research" OR "coolness concept" OR "application of coolness" OR "context of coolness" OR "coolness definition" OR "coolness evaluation" OR "coolness empirical" OR "coolness").

A seleção das fontes de pesquisa foi baseada em duas categorias: periódicos e conferências. Além disto, foram realizadas buscas em referências de teses e dissertações publicados no Brasil e no exterior. Após a definição dos parâmetros de busca, foram realizadas pesquisas em páginas de conferências e periódicos da área de Interação Humano Computador (IHC), ACM Digital Library, IEEE Digital Library, Science Direct, ISI Web of Science, Scopus, Springer Link, Portal Periódicos da CAPES e Google Scholar.

\subsection{Critérios de Inclusão e Exclusão}

Como critério de inclusão foram selecionadas publicações entre janeiro de 2010 a maio de 2020, artigos publicados na língua inglesa ou portuguesa que possuíam um ou mais dos termos de busca pesquisados. Foram excluídos os artigos com informações ou termos da pesquisa duplicadas, sem relevância com os termos pesquisados ou fora do período de publicação definido neste estudo.

\section{Execução da Revisão Sistemática}

A execução da revisão sistemática trata da seleção dos estudos e extração dos dados relevantes para responder às questões de pesquisa e, finalmente, da análise dos dados, que será apresentada na seção de resultados.

\subsection{Seleção dos estudos e extração dos dados}

O processo de pesquisa foi realizado através de uma busca manual de conferências e artigos de periódicos específicos, que foram selecionados por incluírem estudos empíricos ou pesquisas na literatura, ou foram utilizados como fontes para outras revisões sistemáticas da literatura relacionadas às questões de pesquisa. Na organização e registro da pesquisa foi utilizada a ferramenta on-line Parsifal, que possibilitou a importação arquivos de referência, além disso, identificou duplicatas entre as diferentes fontes de pesquisa e possibilitando a avaliação da qualidade das informações durante a extração dos dados.

O processo de seleção dos trabalhos foi realizado em três etapas, sendo na primeira a execução de uma pesquisa com um conjunto de termos específicos em cada biblioteca consultada para encontrar estudos relacionados. Na segunda etapa, foram selecionados os artigos cujos títulos e resumos tiveram maior relevância para realizar a leitura, e rejeitados os duplicados ou que não se encaixavam nos critérios de inclusão e exclusão. Ao final, foram analisados os artigos selecionados, aceitando-os e rejeitandoos com base no conteúdo que cada um possuía ao responder as questões de pesquisa (QP1, QP2). Como resultado do processo de pesquisa, um total de 97 artigos foi identificado inicialmente, a segunda etapa de seleção esse número reduziu para 67 e ao final do processo culminou em 17 artigos aceitos, conforme apresenta a figura 1. 


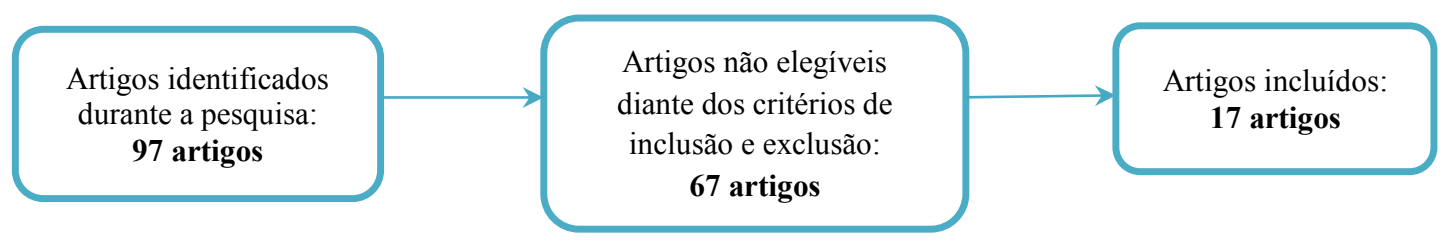

Figura 1. Procedimento para a seleção dos artigos.

\subsection{Resultados}

5.2.1. Respondendo à questão de Pesquisa 1 - Do que se trata o conceito de coolness em produtos digitais?

Os autores descrevem características associadas ao coolness para prover uma noção geral deste conceito. Para entender de que forma o conceito está categorizado em subconceitos, foi sumarizado na tabela 1 as características encontradas nos artigos que estão atreladas ao conceito de coolness.

Tabela 1 - Características associadas ao coolness identificadas pelos autores dos artigos selecionados.

\begin{tabular}{|l|l|}
\hline \multicolumn{1}{|c|}{ Características } & \multicolumn{1}{|c|}{ Autores } \\
\hline Desejabilidade & $\begin{array}{l}\text { Bruun et al. (2016), Raptis et al. (2017), Raptis et al. } \\
\text { (2013), Irshad and Sadiq (2020), Read et al. (2011), } \\
\text { Fitton et al. (2012), Warren and Reimann (2019). }\end{array}$ \\
\hline Usabilidade & $\begin{array}{l}\text { Bruun et al. (2016), Raptis et al. (2017), Raptis et al. } \\
\text { (2013), Irshad and Sadiq (2020), Kim and Park (2019). }\end{array}$ \\
\hline Utilidade & $\begin{array}{l}\text { Holtzblatt (2011), Mamonov and Koufaris (2020), } \\
\text { Farnsworth et al. (2014, June), Kim and Park (2019), } \\
\text { Sundar et al. (2014), Park (2019), Raptis et al. (2017), } \\
\text { Raptis et al. (2013), Irshad and Sadiq (2020). }\end{array}$ \\
\hline Conexão & Holtzblatt (2011) \\
\hline Identidade & $\begin{array}{l}\text { Holtzblatt (2011), Mamonov and Koufaris (2020), } \\
\text { Farnsworth et al. (2014, June). }\end{array}$ \\
\hline Sensação & $\begin{array}{l}\text { Foltzblatt (2011), Mamonov and Koufaris (2020), } \\
\text { Farnsworth et al. (2014, June), Kim and Park (2019) }\end{array}$ \\
\hline
\end{tabular}




\begin{tabular}{|l|l|}
\hline Atratividade & $\begin{array}{l}\text { Kim and Park (2019), Sundar et al. (2014), Park (2019), } \\
\text { Raptis et al. (2017), Raptis et al. (2013), Irshad and } \\
\text { Sadiq (2020), Peng et al. (2016, June), Warren and } \\
\text { Campbell (2014), Farnsworth et al. (2014, June). }\end{array}$ \\
\hline Não convencional & $\begin{array}{l}\text { Kim and Park (2019), Sundar et al. (2014), Park (2019), } \\
\text { Raptis et al. (2017), Raptis et al. (2013), Irshad and } \\
\text { Sadiq (2020), Read et al. (2011), Fitton et al. (2012), } \\
\text { Warren and Reimann (2019), Bruun et al. (2016). }\end{array}$ \\
\hline Inovação & $\begin{array}{l}\text { Kim and Park (2019), Sundar et al. (2014), Park (2019), } \\
\text { Raptis et al. (2017), Raptis et al. (2013), Irshad and } \\
\text { Sadiq (2020), Dar-Nimrod et al. (2012), Peng et al. } \\
\text { (2016, June). }\end{array}$ \\
\hline Rebeldia & $\begin{array}{l}\text { Dar-Nimrod et al. (2012), Read et al. (2012), Raptis et } \\
\text { al. (2013), Bruun et al. (2016), Read et al. (2011), } \\
\text { Fitton et al. (2012). }\end{array}$ \\
\hline Anti-social & $\begin{array}{l}\text { Warren and Reimann (2019), Read et al. (2011), } \\
\text { Fitton et al. (2012). }\end{array}$ \\
\hline
\end{tabular}

\section{Desejabilidade}

Bruun et al. (2016) consideram desejabilidade um dos itens que mede o coolness, estando relacionada à forma como um produto interativo específico agrada ao desejo pessoal, por exemplo, "Este dispositivo pode me fazer feliz" ou "Este dispositivo pode me fazer ficar bem". Read et al. (2012) afirma que o aspecto de um produto ou dispositivo ser muito desejável e inovador, vincula-se às características do dispositivo. Warren and Reimann (2019) destacam que designs incomuns de produtos capturam melhor a atenção do que designs normais, o incomum pode ser considerado legal e desejável desde que faça sentido aos usuários.

\section{Usabilidade}

De acordo com Raptis et al. (2017) a percepção de usabilidade, se refere à aprendizagem, a utilidade e operacionalidade do dispositivo por exemplo, em relação a facilidade de uso. Sundar et al. (2014) apontam que a percepção usabilidade é potencial no julgamento inicial de um produto legal. Kim and Park (2019) descrevem a percepção de usabilidade como padrão-chave de avaliação para o desenvolvimento não empregado em um mercado convencional.

\section{Utilidade}

Mamonov and Koufaris (2020) afirmam que a utilidade é medida através da associação entre a percepção da tecnologia (inovadora/moderna/futurista) e a imagem pessoal esperada, assim como seus benefícios práticos associados ao uso inovador. 
Kim and Park (2019) e Park (2019) definem que a utilidade ou valor utilitário como o nível em que os usuários acreditam que o uso de uma tecnologia específica ajuda no estabelecimento de suas tarefas, aprimorando o desempenho no trabalho e eficiência. Sundar et al. (2014) ressaltam que utilidade ao valor percebido pelo usuário em relação aos produtos adotados que fornecem várias funções e serviços. Raptis et al. (2013) destacam que um produto pode ser considerado legal se for útil para um grupo específico, indicando a filiação a esse grupo em particular.

\section{Conexão}

Para Holtzblatt (2011) a ligação entre pessoas é fundamental para a existência humana. Seja no contexto familiar, social ou profissional, estar com alguém para transcender a solidão é tão necessária quanto a respiração. Nesse sentido os produtos cool ajudam as pessoas a estabelecerem relacionamentos que mais importam.

\section{Identidade}

Mamonov and Koufaris (2020) destacam a associação entre a tecnologia e a imagem pessoal esperada, benefícios práticos vinculados ao uso inovador da tecnologia e sua importância no atendimento das necessidades psicológicas, além das motivações puramente funcionais ou hedônicas. Por exemplo, o ressurgimento do interesse por alguns videogames antigos em novas plataformas como smartphones. Farnsworth et al. (2014, June), Holtzblatt (2011) afirmam que produtos legais nos trazem uma maneira de ver o que os outros da mesma idade ou estágio na vida fazem para se tornar mais adultos, dessa forma nos ajudam a encontrar exemplos de comportamentos, roupas, valores, ou qualquer coisa para ver se parece adequado.

\section{Sensação}

Mamonov and Koufaris (2020) afirmam que a avaliação do quanto um produto afetará a auto-percepção do usuário e as percepções dos outros, são mais importantes que a expectativa de desempenho da tecnologia na consideração da adoção. Para Kim and Park (2019) os produtos que apresentam funções úteis, design moderno e esplêndido são considerados legais, após a aquisição e utilização os usuários se sentem distintos e satisfeitos com a singularidade. Farnsworth et al. (2014, June) e Holtzblatt (2011) destacam a sensação em dois campos: imersão sensorial, criando o "tempo fora do tempo" e momentos de puro prazer sensual, através de produtos (músicas, televisores, jogos, etc.) que oferecem imersões sensoriais, tornando-se o núcleo de nossas atividades de lazer nos absorvendo por longos períodos.

\section{Atratividade}

Sundar et al. (2014) afirmam que a atratividade em produtos interativos abrange tanto o apelo estético quanto uma noção de estilo socialmente aceita. De acordo com Park (2019) sentidos afetivos, como a atratividade, associados aos dispositivos e tecnologias específicas tornam os usuários mais propensos a utilizá-los. Kim and Park (2019) destacam que a atração criada por características estéticas influencia os valores hedônicos e utilitários dos indivíduos quando utilizam um dispositivo ou serviço específico. Para Farnsworth et al. (2014, June) a importância dada a aparência aponta para o fato de que a percepção da estética tem um grande impacto na classificação de produtos. 


\section{Não convencional}

Warren and Reimann (2019) afirmam que a distinção entre o design de produtos legais daqueles que parecem incomuns ou engraçados desviam-se da norma desde que faça sentido, mas sem perturbar o consumidor. Os usuários esperam ser considerados diferenciados através do uso de produtos legais, que projetam suas características e interesses inerentes, aumentando a atitude positiva com relação ao produto [Park 2019].

\section{Inovação}

Park (2019) afirma que produtos exclusivos podem ser sedutores e despertar o interesse dos usuários para obtê-los. Também aponta que os usuários podem obter um senso de identidade e estilo comprando e utilizando mercadorias únicas e exclusivas e exibi-los para os outros verem. Peng et al. (2016, June) referem-se ao grau em que os usuários percebem se uma tecnologia está ausente na cultura convencional, capturada pela avaliação de uma tecnologia, no contexto da aparência, no cumprimento de seu propósito de forma criativa e como permite aos usuários realizarem coisas de uma maneira nova e emocionante. Por exemplo, dispositivos móveis, são percebidos como interessantes pelos jovens por conta de suas características atraentes e originais.

\section{Rebeldia}

De acordo com Dar-Nimrod et al. (2012) e Read et al. (2012) a rebeldia pode ser claramente confirmada como um aspecto da construção, sendo relativamente independente das características convencionalmente desejáveis como: atratividade e simpatia. Raptis et al. (2013) apontam que pessoas consideradas inovadoras ao perceberem um artefato digital como rebelde (exclusivo ou autêntico) com características que tornam o dispositivo fora do comum ou não convencional, logo outras pessoas irão perceber como legal depois de algum tempo. Bruun et al. (2016) sugerem que a rebeldia apresenta construções diferentes das existentes e são indicadas por valores discriminantes e de inconsistência interna.

\section{Anti-social}

De acordo com Warren and Reimann (2019) o design incomum de um produto é considerado legal e desejável quando relacionado a produtos com design humorístico e indesejável, se o desvio da norma faz sentido aos consumidores. Farnsworth et al. (2014, June) afirmam que os aspectos do comportamento anti-social é visto como a chave para ser percebido como legal.

5.2.2 Respondendo à questão de Pesquisa 2 - Como conceito de coolness é operacionalizado para testar o quanto a experiência do usuário está sendo cool em produtos digitais?

Diante da tentativa em definir um instrumento para testar o aspecto de coolness em interfaces com o usuário, cada autor utiliza as características observadas nas áreas de estudo para testar a experiência do usuário operacionalizada por questionários e entrevistas.

O questionário cool desenvolvido por Bruun et al. (2016) apresenta uma proposta validada para medir a percepção interna do coolness. Nesse processo, foram 
identificados 3 fatores relacionados a 17 questões utilizadas especificamente para medir a percepção do coolness interno de um produto interativo. Os participantes avaliaram um dispositivo móvel, respondendo a várias questões através de uma página web, que exibia no lado esquerdo o dispositivo e no lado direito as perguntas. O questionário mediu a conveniência através de questões relacionadas à forma como um produto interativo específico pode apelar para o desejo pessoal.

Warren and Campbell (2014) investigaram a relação entre o coolness e o conceito de autonomia, e como os fatores que podem afetá-lo. Foram recrutados nessa pesquisa, 190 participantes residentes nos Estados Unidos, que leram informações a respeito de um varejista de café, de uma marca familiar e outra desconhecida, estavam alterando o design de suas garrafas de água e tiveram acesso a um item de cada garrafa com os novos logotipos. Os participantes relacionaram a percepção de coolness ao completarem duas escalas relacionadas com não legal ou legal de acordo com as perguntas do questionário. Como resultado da pesquisa, foi verificado um consenso geral sobre a percepção do que é legal no contexto fornecido.

Read et al. (2011) realizaram uma pesquisa com adolescentes de uma escola do Reino Unido, com idades entre 11 e 15 anos, cursando o $7^{\circ}$ ano e $10^{\circ}$ ano. Eles foram instruídos a criar, no contexto de seus quartos, "a vida que gostariam de ter", sem muita fantasia. $\mathrm{O}$ estudo apresentou diferenças nas percepções de cool entre os sexos e idades.

Read et al. (2012) realizaram estudos concentrando em dividir as características em entidades menores e utilizá-los sistematicamente na construção de blocos para produzir um questionário com foco nos domínios de interação das áreas de design, marketing e indústria da música/cinema com intuito de aferir a percepção do coolness em um produto. Através de uma ferramenta visual interativa, o protótipo de um aplicativo denominado Cool Wall, proporciona que imagens possam ser classificadas em quatro categorias ('sério não legal', 'não legal', 'legal' e 'abaixo de zero'). O resultado do estudo mostra que os itens desejáveis foram classificados como legais, as tecnologias móveis mais caras como itens mais legais, seguidos de itens alimentares que seriam considerados não saudáveis pelos pais dos alunos (doces e fast food).

Farnsworth et al. (2014) queriam entender o que era essencial para a experiência cool do usuário e associá-lo para que pudesse ser usada sistematicamente para desenvolver produtos transformadores, pois acreditam que com o entendimento dos princípios subjacentes que tornam um produto interessante, eles poderiam ser utilizados para projetar deliberadamente um produto legal.

A pesquisa do consumidor - Verão 2010, contou com o envolvimento de 65 consumidores americanos com idades entre 15 e 60 anos. As entrevistas foram presenciais, realizadas na casa dos participantes que discutiram e responderam 3 perguntas. Como resultado, foi gerado um diagrama de afinidade contendo os dados qualitativos dos principais temas (construções) que definiram a estrutura conceitual originando sete conceitos: quatro (realização, conexão, identidade e sensação) definiram a maneira como o produto gera alegria, afetando a vida das pessoas e cumprindo seus principais desejos, e três (direto à ação, fator de aborrecimento e delta do aprendizado) definiram a maneira como as pessoas experimentam e utilizam o próprio produto. 
$\mathrm{Na}$ pesquisa Smart $T V s$, os autores utilizaram questionários que incluíam perguntas a partir de estudos anteriores acerca de itens sobre utilidade, apelo subcultural, originalidade, atratividade, atitude, e intenção de uso. Em seguida, especialistas em radiodifusão e tecnologias de mídia realizaram a revisão e correção dos itens do questionário, em seguida foi realizada uma pesquisa piloto com 20 participantes que possuíam mais de 6 meses de experiência no uso de TVs inteligentes.

Os estudos apresentados utilizaram questionários e entrevistas para testar a experiência do usuário além de medir a percepção do coolness nas áreas de IHC e no design de interação. O questionário produzido por Bruun et al. (2016), denominado "O questionário cool", mediu a conveniência através de questões relacionadas à forma como um específico produto interativo, representado por um dispositivo móvel apela para o desejo pessoal. Através de uma página web, os participantes responderam perguntas relacionadas ao dispositivo, e a partir desse questionário foram extraídas as características identificadas pelos usuários como, por exemplo, a rebeldia, que pode ser medida através de declarações relacionadas à convencionalidade do dispositivo.

Warren and Campbell (2014) investigaram a relação entre o coolness e o conceito de autonomia, descrevendo como os fatores que podem afetá-lo e como a autonomia pode ser medida através de um questionário. Os estudos foram realizados com participantes que avaliaram design de garrafas e logotipos de marcas famosas, onde concluíram que tanto marcas quanto objetos divergem na percepção do usuário aumentando a autonomia de uma maneira apropriada e percebida como cool.

$\mathrm{Na}$ pesquisa realizada, Read et al. (2011) buscaram entender elementos de design voltados para adolescentes que pudessem ser identificados como legal, e quais seriam suas causas e motivações. Definiram características com base em pesquisas na literatura e trataram categorias específicas (retrô, autêntico, alto valor, rebelde, antisocial e inovador) para checar as informações extraídas a partir de respostas dos alunos de uma escola do Reino Unido, com idades entre 11 e 15 anos. Como resultado, foram observadas características especiais em relação ao design, marcas e especificações de produtos que foram associadas entre as categorias.

Relacionado com um trabalho anterior [Read et al. 2011] Read et al. (2012) concentraram na premissa de que existe um entendimento comum entre adolescentes do que é legal, assim com a expectativa que pode haver algumas "coisas" que seriam consideradas menos ou mais legais por certos subgrupos. "Coisas" referem-se a objetos físicos (um tipo de tecnologia) ou outros itens físicos que escolhemos consumir (um determinado tipo de alimento) ou associar. Para avaliar esse entendimento, foi desenvolvido um aplicativo, o Cool Wall, que apresenta imagens exibidas em uma sequência predefinida, e ao serem arrastadas até uma categoria desejada às classificações definidas pelo usuário são armazenadas em um arquivo.

Entrevistas também foram utilizadas nesse processo, Farnsworth et al. (2014) desenvolveram um questionário para medir o coolness, a partir da realização de vários estudos durante três anos, envolvendo 900 consumidores e mais de 2000 profissionais de negócios de todo o mundo. Como resultado, as principais características descritas pelos entrevistados contribuíram para definir estrutura conceitual do coolness. 


\section{Conclusões}

Tradicionalmente o termo cool é uma forma com que as pessoas costumam referenciar positivamente objetos, atividades, pessoas ou lugares. Em áreas como marketing, indústrias da moda e música, o coolness é descrito como uma boa atitude em relação a algo e como as pessoas o reconhecem, sendo sua percepção compartilhada entre os membros de um grupo. O conceito de coolness vem sendo cada vez mais utilizado para descrever a experiência e aprovação dos usuários em vários produtos interativos em IHC. Contudo, apesar de ser um critério evidente para novos dispositivos e aplicações, esse é um conceito complexo que pode significar coisas diferentes entre as pessoas.

Na presente revisão sistemática da literatura, buscamos estudos que trataram o conceito de coolness na área de IHC, com o objetivo de identificar suas características fundamentais e prover uma noção ampla do termo. A realização desse trabalho proveu uma visão mais abrangente do conceito do coolness envolvendo as características destacadas na literatura. A subconceitualização de coolness pode ser útil para pesquisadores e projetistas desenvolverem projetos almejando uma melhor UX. Desse modo, apresentando uma contribuição para a área de IHC, possibilitando, a partir deste estudo, o surgimento de novas ideias sobre como projetar artefatos legais, evocando uma melhor experiência do usuário.

Esta pesquisa desvendou que o coolness pode ser considerado uma atitude pessoal em relação a objetos, pessoas e atividades, que são caracterizados de acordo com a percepção do usuário acerca dos fatores definidos nas características. Além disso, nosso estudo pode ser utilizado como ponto de partida para o desenvolvimento de questionários e outros instrumentos para medição do quanto um produto digital é experienciado como cool. Nesse contexto, fornecemos exemplos práticos tanto na identificação de como os artefatos são percebidos como cool, quanto sobre como utilizar os resultados coletados a partir de ferramentas utilizadas para medir a percepção de coolness.

Outra contribuição dessa revisão sistemática da literatura foi desvendar como está sendo testado, diante da experiência e aprovação dos usuários em relação a várias características, o quanto um produto é definido como cool. Diante dos conceitos apresentados, podemos inferir que não se pode medir diretamente o coolness de um objeto, mas podemos medir o nível de percepção das pessoas baseadas em valores pessoais, criados e abordados de acordo com os grupos pertencentes.

No entanto, foi identificada uma lacuna na literatura no que diz respeito a uma ferramenta mais abrangente para testar o coolness em UX. Apenas foram encontradas ferramentas limitadas que não abordam todos os conceitos supracitados. Muitos autores realizaram estudos baseados em questionários e entrevistas, e em alguns casos, selecionaram algumas características já identificadas na literatura para a realização estudos, visando relacionar as características ou propondo novas categorizações, apontando a necessidade de pesquisas nesse sentido.

A presente revisão sistemática da literatura servirá como ponto inicial para investigações acerca de como cada subconceito do coolness, pode ser observado em websites e aplicativos e se eles podem, efetivamente, ser atrelados à esse conceito nesse 
cenário específico. Em outras palavras, como as características vinculadas ao conceito coolness, provenientes da literatura, desejabilidade, utilidade, usabilidade, conexão, identidade, sensação, atratividade, não-convencional, inovação, rebeldia e anti-social, podem ser mapeadas em exemplos concretos de websites e aplicativos e se podem ser usadas para se julgar se um website ou aplicativo é legal. Para tanto serão realizados testes empíricos envolvendo websites e aplicativos considerados cool e não cool, considerando-se um número significativo de usuários, buscando prover evidências que indiquem se as características compiladas na presente pesquisa estão relacionadas ao coolness nessa perspectiva.

\section{Referências}

Brereton, Pearl et al. (2007). Lessons from applying the systematic literature review process within the software engineering domain. Journal of systems and software, v.80, n.4, p.571-583.

Bruun, Anders et al. (2016). Measuring the coolness of interactive products: The COOL questionnaire. Behaviour \& Information Technology, v.35, n.3, p. 233-249.

Dar-Nimrod, Ilan et al. (2012). Coolness: An empirical investigation. Journal of Individual Differences.

Farnsworth, Carol et al. (2014). Cool in Business: Developing a Data-Based Instrument Measuring "Cool". In: International Conference of Design, User Experience, and Usability. Springer, Cham, p. 232-243.

Farnsworth, Carol et al. (2014). Measuring product" coolness" developing a measurement instrument. In: CHI'14 Extended Abstracts on Human Factors in Computing Systems, p. 893-896.

Fitton, Daniel et al. (2012). Constructing the cool wall: a tool to explore teen meanings of cool. PsychNology, v. 10, n. 2, p. 141-162.

Hassenzahl, Marc; Tractinsky, Noam. (2006). User experience - a research agenda. Behaviour \& information technology, v. 25, n. 2, p. 91-97.

Holtzblatt, Karen; Rondeau, David B.; Holtzblatt, Les. (2010). Understanding" cool". In: CHI'10 Extended Abstracts on Human Factors in Computing Systems, p. 3159-3162.

Holtzblatt, Karen. (2011). What makes things cool? Intentional design for innovation. Interactions, v.18, n. 6, p.40-47.

Irshad, Shahida; Sadiq, Mazhar. (2020). Measuring The Coolness Of Pakistani Truck Art Emojis. International Journal of Computer Science and Information Security (IJCSIS), v.18, n.3.

Jetter, Christian; Gerken, Jens. (2007). A simplified model of user experience for practical application. In: NordiCHI 2006, Oslo: The 2nd COST294-MAUSE International Open Workshop "User eXperience-Towards a unified view", p. 106-111.

Kim, Jina; Park, Eunil. (2019). Beyond coolness: Predicting the technology adoption of interactive wearable devices. Journal of Retailing and Consumer Services, v.49. 
Mamonov, Stanislav; Koufaris, Marios. (2020). Fulfillment of higher-order psychological needs through technology: The case of smart thermostats. International Journal of Information Management, v. 52, p. 102091.

Mccrickard, D. Scott et al. (2012). Understanding Cool in Computing for AfricanAmerican Youth.

Nancarrow, Clive; Nancarrow, Pamela; Page, Julie. (2002). An analysis of the concept of cool and its marketing implications. Journal of Consumer Behaviour: An International Research Review, v. 1, n. 4, p. 311-322.

O'Donnell, Kathleen A.; Wardlow, Daniel L. (2000). A Theory on the Origins of Coolness. ACR North American Advances.

Park, Eunil. Understanding the social adoption of smart TVs: the key role of product coolness. (2019) Universal Access in the Information Society, p. 1-8.

Peng, Xixian; Zhao, Yuxiang Chris; Teo, Hock-Hai. (2016). Understanding Young People's Use of Danmaku Websites: the effect of Perceived Coolness and subcultural Identi. In: PACIS, p. 252.

Pountain, Dick et al. (2000). Cool rules: Anatomy of an attitude. Reaktion books.

Price, Linda L.; Belk, Russell W. (2016). Consumer ownership and sharing: Introduction to the issue. Journal of the Association for Consumer Research, v. 1, n. 2, p. 193-197.

Raptis, Dimitrios; Kjeldskov, Jesper; Skov, Mikael. (2013). Understanding "cool" in human-computer interaction research and design. In: Proceedings of the 25th Australian Computer-Human Interaction Conference: Augmentation, Application, Innovation, Collaboration, p. 53-62.

Raptis, Dimitrios et al. (2017). Converging coolness and investigating its relation to user experience. Behaviour \& Information Technology, v. 36, n. 4, p. 333-350.

Read, Janet et al. (2011). Understanding and designing cool technologies for teenagers. In: CHI'11 Extended Abstracts on Human Factors in Computing Systems, p. 1567-1572.

Read, Janet C. et al. (2012). Cool across continents, cultures and communities. In: CHI'12 Extended Abstracts on Human Factors in Computing Systems, p. 2791-2794.

Southgate, Nick. (2003). Coolhunting, account planning and the ancient cool of Aristotle. Marketing Intelligence \& Planning.

Sundar, S. Shyam; Tamul, Daniel J.; Wu, Mu. (2014). Capturing “cool”: Measures for assessing coolness of technological products. International Journal of HumanComputer Studies, v. 72, n. 2, p. 169-180.

Warren, Caleb; Campbell, Margaret C. (2014). What makes things cool? How autonomy influences perceived coolness. Journal of Consumer Research, v. 41, n. 2, p. 543-563.

Warren, Caleb; Reimann, Martin. (2019). Crazy-Funny-Cool Theory: Divergent Reactions to Unusual Product Designs. Journal of the Association for Consumer Research, v. 4, n. 4, p. 409-421. 